\title{
Case report: laparoscopic radical cystectomy on a patient with Behçet's syndrome, our initial experience on perioperative safety
}

\author{
Xiaoqiang Xue ${ }^{1 \#}$, Xinyi Yang ${ }^{2 \#}$, Zhigang $\mathrm{Ji}^{1}$, Yi Xie ${ }^{1}$ \\ ${ }^{1}$ Department of Urology, Peking Union Medical College Hospital, Chinese Academy of Medical Sciences and Peking Union Medical College, \\ Beijing, China; ${ }^{2}$ Department of Health Care, Peking Union Medical College Hospital, Chinese Academy of Medical Sciences and Peking Union \\ Medical College, Beijing, China \\ \#These authors contributed equally to this work and should be regarded as co-first authors. \\ Correspondence to: Yi Xie; Zhigang Ji. Peking Union Medical College Hospital, Chinese Academy of Medical Sciences and Peking Union Medical \\ College, No.1 Shuaifuyuan Road, Dongcheng, Beijing 100730, China. Email: xieyi6262@163.com; jizhiganguro@126.com.
}

\begin{abstract}
Behçet's syndrome (BS) is a rare systemic vasculitis that involves multiple systems and organs. Owing to the long-term prescription of immunosuppressive drugs, patients with BS are prone to urothelial tumors. However, surgical treatment in these cases could be dangerous because of the potential BS-related vasculitis, bleeding, and anastomotic necrosis. Literature reported that only five radical cystectomy cases had been performed on patients with BS, whereas the perioperative mortality was $40 \%$. Moreover, perioperative managements on these cases were less discussed due to their multidisciplinary nature and rarity. We herein report a 54-year-old patient with a 13 -year history of BS, diagnosed with sarcomatoid urothelial carcinoma and successfully underwent laparoscopic radical cystectomy with bilateral ureterocutaneostomy. No evidence of tumor residual or ureterocutaneostomic necrosis was reported in the subsequent three months of followup. In this case, the advantages of minimally invasive surgery in dealing with the parailiac lymph nodes were demonstrated. This article presented our thoughts, strategies, and experience on ensuring the patient's perioperative safety, as well as the selection of urinary diversion. We reviewed the literature in both English and Mandarin Chinese. Currently, two published articles describe the adoption of cystectomy in treating bladder cancer patients complicated with BS, whereas not any had shared the experience of using minimally invasive surgery. Hopefully, it could offer updated and enlightening significance to all related medical practitioners.
\end{abstract}

Keywords: Bv (BS); laparoscopic radical cystectomy; case report; surgical management; perioperative safety

Submitted Nov 12, 2021. Accepted for publication Dec 09, 2021.

doi: $10.21037 / \mathrm{tau}-21-1011$

View this article at: https://dx.doi.org/10.21037/tau-21-1011

\section{Introduction}

First introduced in 1937, Behçet's syndrome (BS) is an unexplained rare systemic variable vessel vasculitis that involves the skin, mucosa, eyes, joints, arteriovenous system, nervous system, and the gastrointestinal system (1). Malignant tumors have been sporadically reported in patients with BS. The hypothesis suggested it might be the immunosuppressants or the autoimmune process per se that induced the carcinogenesis $(1,2)$. The proportion of solid neoplasms varied amongst all reported malignancies, while a fraction was on the bladder $(3,4)$. On the other hand, radical cystectomy with urinary diversion has become a standard treatment for advanced bladder cancer (5). To our knowledge, only five cases of radical cystectomy complicated with BS had been reported $(6,7)$. The average blood loss was over $1,500 \mathrm{~mL}$, and the mortality was $40 \%$.

Here, we report our initial experience of laparoscopic radical cystectomy on a BS patient with bladder cancer. 
This article showcases the feasibility of performing minimally invasive surgery on BS patients with urinary tumors and shares our thoughts, strategies, and experience on ensuing their perioperative safety. We present the following article in accordance with the CARE reporting checklist (available at https://tau.amegroups.com/article/ view/10.21037/tau-21-1011/rc).

\section{Case presentation}

A 54-year-old male had been presented with intermittent hematuria for five years. He refused to take thorough examinations until the symptom tended to be uncontrollable in December 2020. After ultrasound confirmed the presence of multiple neoplasms in his bladder, the patient underwent transurethral resection of bladder tumor (TURBt) in January 2021. While he was receiving intravesical chemotherapy, several neoplasms recurred on his left bladder wall, and a second-time TURBt was performed four months later. The final pathological diagnosis was sarcomatoid urothelial carcinoma, no myometrial infiltration was found in the TURBt specimen.

In the aspect of comorbidities, Behçet's syndrome was first diagnosed in 2008 with typical manifestations including recurrent oral and genital ulcers, arthritis, increased high sensitivity C-reactive protein (hsCRP), and an accelerated erythrocyte sedimentation rate (ESR). Cyclophosphamide (CTX) and cyclosporine (CsA) have been prescribed since then for immunosuppressive purposes. BS-related uveitis and blindness occurred in 2016. CTX and CsA were ceased since January 2021 because of the discovery of bladder neoplasms. By the time of his visit to the outpatient clinic, he was on 10 or $15 \mathrm{mg}$ of methylprednisolone alternately every other day. Other comorbidities included hypertension and impaired glucose tolerance, were under proper control.

As sarcomatoid urothelial carcinoma is a rare but aggressive variant of bladder cancers (8), radical surgery was recommended. A multidisciplinary task force led by urologists was established, joined by rheumatologists, anesthesiologists, scrub nurses, and vascular surgeons. $50 \mathrm{mg}$ of hydrocortisone succinate was intravenously dripped every 12 hours during his perioperative fasting and water deprivation.

Considering the large incision of traditional radical cystectomy and poor wound healing ability in patients with BS, we decided to perform the cystectomy minimalinvasively. In case of uncontrollable bleeding and midway laparotomy, we compromised with laparoscopic radical cystectomy rather than robot-assisted laparoscopic radical cystectomy. The operation of laparoscopic radical cystectomy with standard pelvic lymph node resection and bilateral ureterocutaneostomy was performed in August. Intraoperative exploration showed that the patient had thin visceral fat tissue, proliferative pelvic vessels, and enlarged parailiac lymph nodes. Therefore, the surgical team made three core principles accordingly: (I) in case of anastomotic necrosis, we preferred to adopt a simple, less extensive but easy-observative urinary diversion method: bilateral ureterocutaneostomy; (II) attention should be paid to unnecessary clamping, stretching, or sharp stabbing made by dissecting forceps; (III) to avoid postoperative bleeding and reduce the amount of drainage, the coagulation feature of ultrasonic scalpel shall be used as often as possible when facing unclear vessel-like tissue, and Hem-O-Lock clamps shall also be used if necessary.

The operation lasted for 305 minutes, and intraoperative blood loss was $200 \mathrm{~mL}$. The patient was transferred to the general ward afterward. The patient started early ambulation on postoperative day one. The results of ESR, D-dimer, and hsCRP were routinely monitored. He resumed oral methylprednisolone intaking on postoperative day three. No necrosis or delayed bleeding was found on the incision and bilateral ureterocutaneostomas (Figure 1). The patient was discharged on postoperative day 12 .

The pathological result indicated that proliferative spindle cells could be seen in the full thickness of the left bladder wall. No tumor residual, lymphatic vessels invasion or metastatic lymph node was found (0 out of 10). Immunohistochemical result: CK14 (-), CK 20 (-), CK5/6 (-), Desmin (+), GATA3 (-), Her-2 (0), Ki-67 (index $5 \%)$, P53 (-), AE1/AE3 (+). The final TNM stage was TONOM0. We discussed this report with our colleague in the Department of Pathology. They suggested the presence of proliferative spindle cells could be seen as evidence of the previous existence of sarcomatoid urothelial carcinoma. In contrast, the tumor might have been entirely resected in the last TURBt operation. Yet, this result is not predictable unless we have the entire specimens. No evidence of tumor residual or ureterocutaneostomic necrosis was found on his latest follow-up visit in December 2021 (Figure 2).

The patient and his family members are satisfied with the treatment plan when asked on his latest follow-up. This is mainly due to sufficient preoperative education and successful surgical operation. However, because of the extinct nature of ureterocutaneostomy, this patient must replace his ureteral stents regularly. We could sense the patient's complaints about it (usually every two to three 

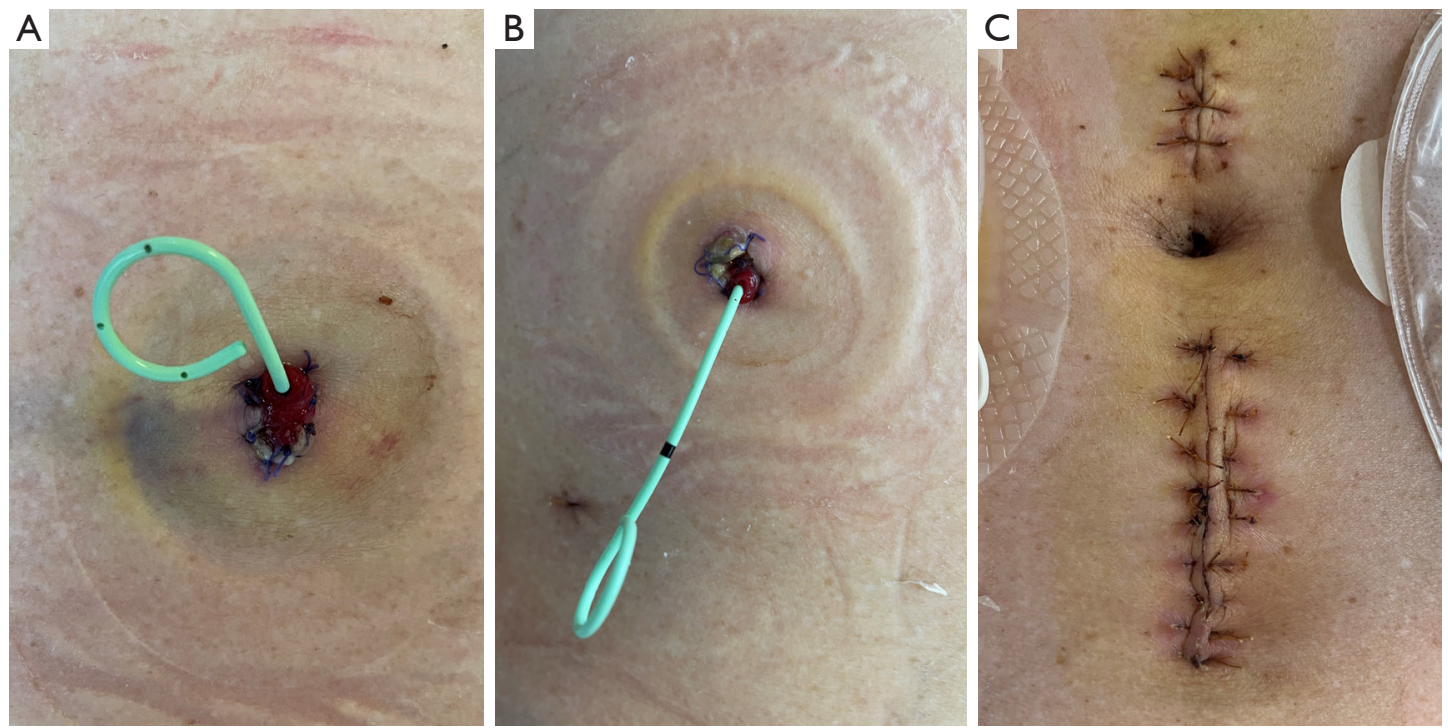

Figure 1 Postoperative view of bilateral ureterocutaneostoma and wound healing. (A) Blood supply of the left ureterocutaneostoma on postoperative day 12; (B) blood supply of the right ureterocutaneostoma on postoperative day 12; (C) no wound infection, bleeding, or dehiscence on the discharging day.
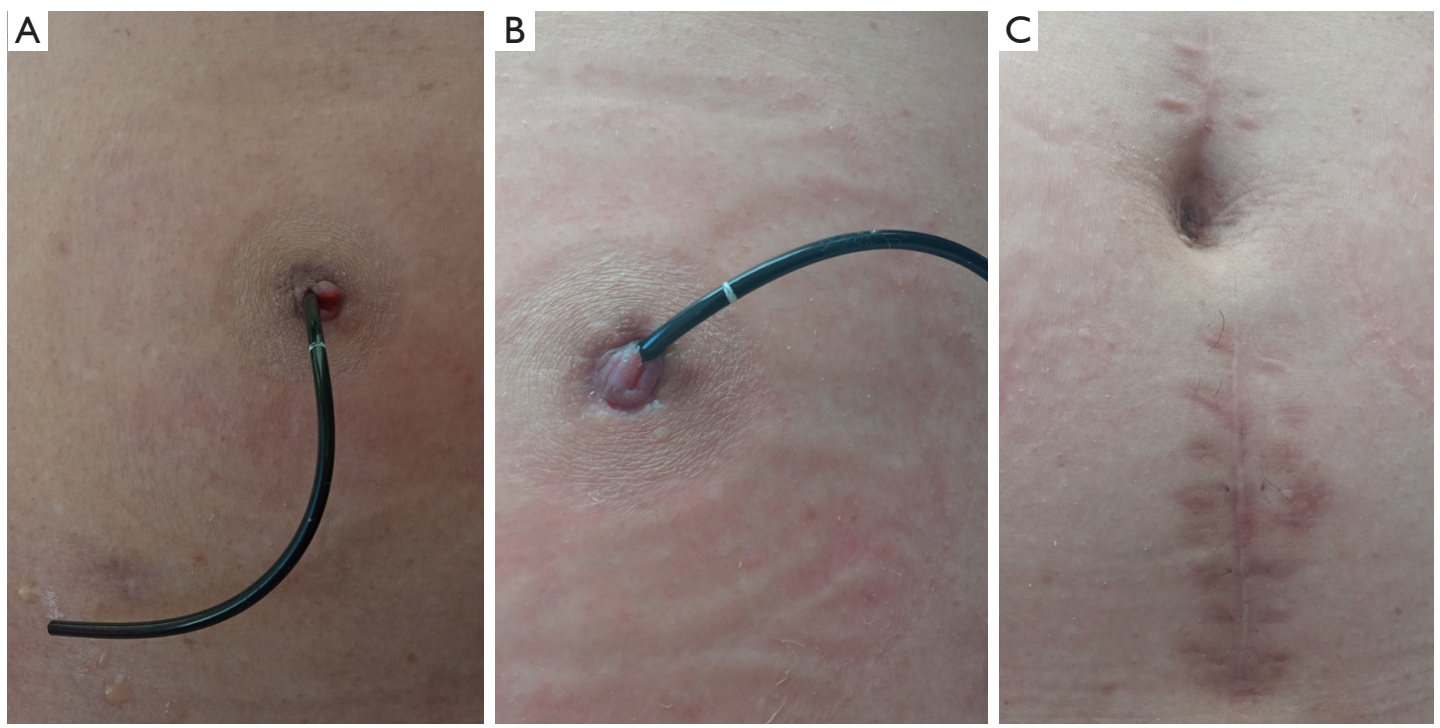

Figure 2 Four-month follow up view of bilateral ureterocutaneostoma and wound healing. (A) Blood supply of the left ureterocutaneostoma four months after the operation; (B) blood supply of the right ureterocutaneostoma four months after the operation; (C) no wound infection, bleeding, or dehiscence four months after the operation.

months), especially during the coronavirus pandemic. Finally, we could also tell that he did not want to make too many comments on his erectile function when interviewed. Hopefully, with the accumulation of experience, we could endeavor urinary diversion techniques that do not need regular ureteral stents replacement, such as orthotopic neobladder.

All procedures performed in this study were in accordance with the ethical standards of the institutional and/or national research committee(s) and with the Helsinki Declaration (as revised in 2013). Written informed consent was obtained from the patient for publication of this case 
report and accompanying images. A copy of the written consent is available for review by the editorial office of this journal.

\section{Discussion}

\section{The relationship between bladder cancer and CTX}

Understanding the relationship between bladder cancer and CTX is in a spiral process. Acrolein, a metabolite of CTX, was reported to be urotoxic in 1979 (9). Eight years later, a cohort of 119 patients who adopted cyclophosphamide for treating rheumatoid arthritis was introduced, the incidence rate of bladder cancer in that cohort was $5 \%(10)$. Moreover, half of the tumors were diagnosed after eleven years or later. Similarly, in 1991, Sigal et al. (11) revealed the time of initial tumor occurrence was between 15 to 180 months. Those studies also suggested bladder cancer might not be associated with the daily intake of CTX. In contrast, it was related to the cumulative dosage, that is, the greater the amount of CTX is, the higher the risk of tumor occurrence would be, and this risk could last for several years after drug withdrawal $(10,11)$.

As in this case, the patient started CTX treatment in 2008 (100 mg/day), and the prescription was alternated to $100 \mathrm{mg}$ of CTX every other day in 2016. The drug was ceased in 2021 owing to the discovery of bladder neoplasms. Hence, the total CTX intake was estimated to be 350-383 grams. This the time of tumor occurrence and the cumulative amount of CTX intake are in line with previously published articles $(10,11)$.

\section{Preoperative preparations, examinations, and the best time for surgery}

Several point-based evaluating forms have been introduced to assess the activity of BS (12-14). Some scales are based purely on BS symptoms per se, while others also include laboratory examination results. Observative indices that revile the activity of BS include oral and genetic ulcers, ocular symptoms, skin lesions, joint pain/arthritis, vomiting/ abdominal pain, diarrhea with bloody stool, and elevation of ESR $(>20 \mathrm{~mm})$ and CRP $(>8 \mathrm{mg} / \mathrm{L})$. However, the disease severity should be assessed by experienced rheumatologists as specific cut-off values to define the stage of BS are not currently available $(12,13)$. In Japan, the activity of BS is determined to be active if a patient presents two or more major evaluating criteria (12). Involvement of the ocular, vascular, neurological, and gastrointestinal systems might be associated with poor prognosis (1). For well-controlled $\mathrm{BS}$, we recommend that the surgery be planned according to the patient's age, gender, types of organ involvement, and oncological prognosis.

Published data suggested that the incidence of vascular lesions in patients with BS was about $25-37 \%$, lesions were mainly deep vein thrombosis, aneurysm, obliterative vasculitis, and arteritis $(15,16)$. As a result, a thorough rheumatological evaluation was recommended. In this patient, echocardiography was conducted to assess the cardiac ejection function, determining cardiac valvular lesions and thrombi. CT angiography or ultrasound of the lower extremity vessels is also recommended. It helps in detecting tortuous vessels and excluding aneurysms, reducing the chances of intraoperative bleeding during pelvic lymph node resection. Inflammatory indicators such as ESR and hsCPR should be routinely monitored. Note that these evaluations cannot determine whether the patient is free of risks. It could only confirm those unprepared.

To date, only five cases of radical cystectomy complicated with BS have been reported $(6,7)$. A detailed comparison between our patient and the other 4 cases (one with missing data) can be seen in Table 1. The conclusion could be drawn that spontaneous bleeding was the most lethal complication. We noticed that colchicine was used for anti-inflammation purposes in all other cases. The author indicated that he abandoned immunosuppressants because they might have potential carcinogenic effects (7). However, we doubted the rationality of abdicating cyclophosphamide and cyclosporine as the European League Against Rheumatism (EULAR) Standing Committee for Clinical Affairs recommended immunosuppressants for treating BS-related arterial involvement. At the same time, colchicine was suggested as the initial treatment in BS patients with acute arthritis (1). The application of colchicine alone might help relieve joint pain and alleviate inflammatory factors, whereas arteritis is continued and irreversible. Specific to individual cases, those four patients might be more vulnerable and less prepared, which might offer a clue to the unknown spontaneous bleeding.

With the help of long-term usage of glucocorticoids and immunosuppressants, the patient's ESR and hsCRP were within the normal range. He reported no ongoing ulcers or arthritis symptoms, indicating a remitting stage of BS. Intraoperative exploration showed no severe inflammatory reaction on major vessels' vascular sheath and adventitia. Perioperative glucocorticoids treatment should be adhered 


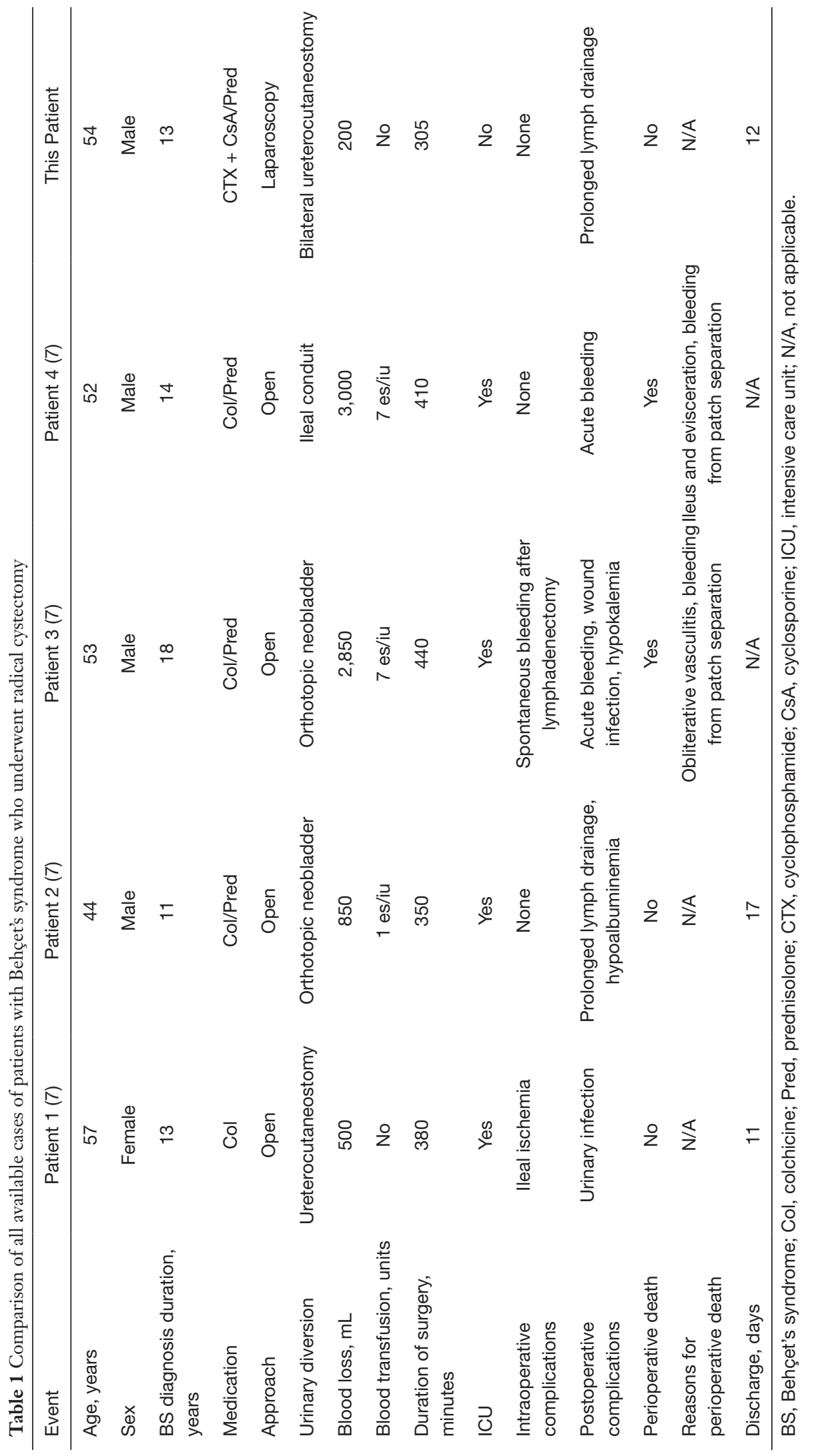



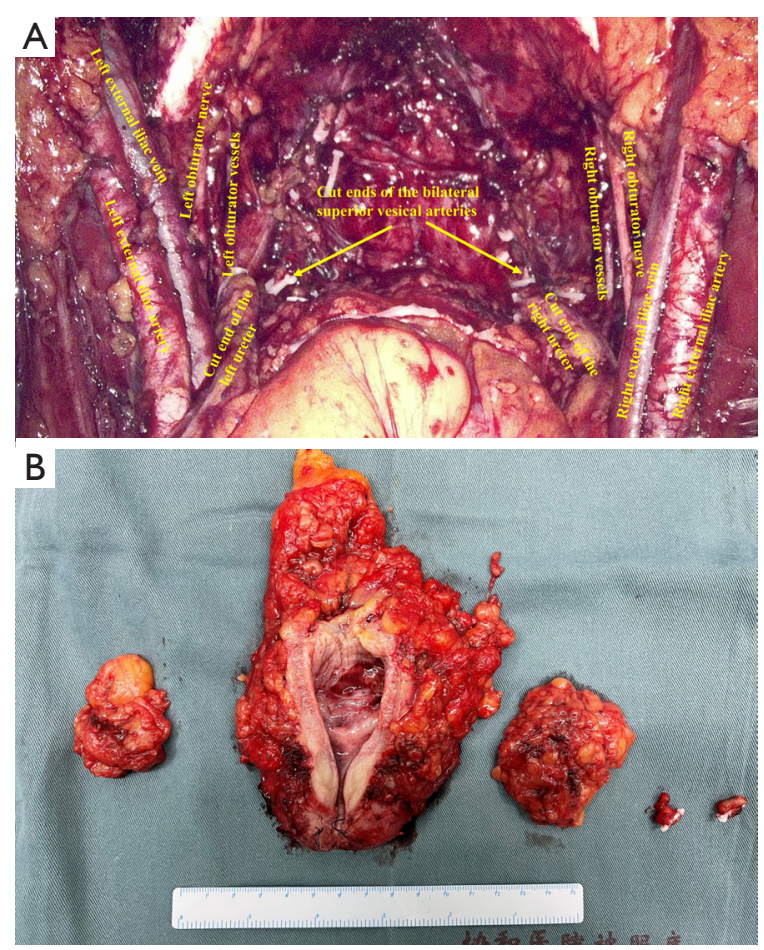

Figure 3 Intraoperative views and gross pathology view. (A) No vessel damage after the complete resection of the bladder, prostate, and bilateral parailiac lymph nodes; (B) gross pathology, from left to right: the left parailiac lymph nodes; the bladder, its adjacent prostate (behind the bladder), and bilateral seminal vesicles (behind the bladder); the right parailiac lymph nodes; the cut end of the left ureter; the cut end of the right ureter.

to as it might be helpful to stabilize the inflammatory response and decrease the incidence of postoperative anastomotic complications $(1,16)$.

\section{Our thoughts on urinary diversions, bowel preparations, and surgical approaches}

Previous studies indicated that BS could involve the mesenteric vessels and cause chronic ischemic inflammation on the intestinal wall. In contrast, around two-third of the patient might not have apparent symptoms of active bleeding and gastrointestinal ulcers $(1,17)$. Therefore, the confirmation of gastrointestinal involvement of BS is complicated (1). Owing to its rarity, experience on BS patients undergoing gastrointestinal surgery is lacking. Sevinc et al. (7). reported a spontaneous ileal ischemia case who had no gastrointestinal complaints nor any definite ulcer foci found in the gross pathology. The author concluded that notwithstanding long-term immunosuppressants or steroids prescription, chronic arteritis could gradually and ultimately cause intestinal ischemia $(7,18)$. We consulted our colleagues from the Department of General Surgery, who opined that whether the anastomotic leakage would happen or not majorly depended on three factors: the blood supply of the anastomosis, regional tension, and stitching techniques. For patients with BS, they would suggest open anastomosis, when necessary, because it could provide surgeons with an intuitive feeling of the anastomotic tension and opportunity of serosal-muscle layer embedding, and reduce chances of impairing major vascular arches. Overall, urinary diversion and bowel preparation methods should be considered according to the patient's situation.

Since this patient was blind, the appeal of self-care was not of primary consideration. Ureterocutaneostomy was preferred in the case of BS-induced postoperative intestinal anastomosis fistula. In the case of the ischemic necrosis of the ureterocutaneostoma, we performed the ureterocutaneostomy bilaterally for double insurance. As urinary diversion was relatively more uncomplicated, the average operation time was shortened by 85 minutes.

We suggested a simplified bowel preparation as an excessive one might cause intestinal flora disorder and potential postoperative ileus and intestinal mucosa lesions and, after that, digestive tract ulcers $(1,19)$.

To reduce intraoperative bleeding, we preferred miniinvasive approaches such as laparoscopic radical cystectomy (LRC) or robot-assisted LRC. They could provide a clear view and magnified vision for recognizing small blood vessels. However, due to its rarity, there was no published data on the superiority between mini-invasive surgery and open surgery on BS patients, and this was barely our primary thought. Different surgeons might choose approaches they are best at according to their preferences, habits, and patients' conditions.

As most delayed bleeding was caused by intraoperative damage and graft detachment, a core of the operation technique was to be gentle and avoid excessive traction. Metastatic lymph nodes could be treated by postoperative chemotherapy or immunotherapy. A balance is needed to be reached between the risk of dissection bleeding and lymph nodes residue (Figure 3). On account of a small incision, it should theoretically reduce the possibility of poor wound healing. Wound dressing with fibroblast growth factors 
solution was also used in this case.

\section{Postoperative monitoring and patient education}

The role of postoperative monitoring and patient education cannot be ignored. Inflammatory evidence such as ESR, hsCRP, ulcers, and wound oozing should be regularly checked. As the patient was blind and did not exercise too much, he was encouraged to start early ambulation under his wife's custody. This would promote gastrointestinal peristalsis, establish self-confidence, avoid decubitus and prevent deep venous thrombosis.

To avoid intraoperative gastrointestinal flatulence, it is necessary to indwell the gastric tube after anesthesia. The gastric tube could be withdrawn if there is not much gastric juice postoperatively. The time of drainage tube withdrawing is not different from ordinary cases. Acid suppressants are recommended in case of stress ulcers. Since the intestine was intact in this patient, oral food-intaking, including the prescription of glucocorticoids, was resumed on postoperative day three. To prevent postoperative ileus and its following flatulence and intestinal ischemia, the patient was recommended to prophylactically take $20 \mathrm{~mL}$ of liquid paraffin oil daily and chew gum. Paraffin oil is rarely absorbable and non-digestible, colorless and tasteless mineral oil, preventing water absorption, lubricating the intestinal wall and feces, and softening the stool (19). Chewing gum can promote gastrointestinal peristalsis and digestive enzyme secretion (20).

Two limitations should be noted. That is, on account of the rarity nature of this case, more evidence should be collected comprehensively. Moreover, longer-term outcomes are to be seen by expanding the follow-up time.

\section{Conclusions}

With the prevalence of immunosuppressants, more and more urothelial malignancies might be found in patients with BS. Thorough preoperative evaluations, including inflammatory indices, radiological imaging, and physical examinations, could help exclude those who are not prepared. Rare and risky as BS is, under the premise of well preoperative drug prescription and complete blood vessels ligation, it is not an absolute contraindication of radical cystectomy. Mini-invasive surgery could help to identify blood vessels better. Urinary diversion should base on the patient's condition and gastrointestinal function. Non-interrupted perioperative use of glucocorticoids, close postoperative monitoring, and patient education are recommended.

\section{Acknowledgments}

Funding: None.

\section{Footnote}

Reporting Checklist: The authors have completed the CARE reporting checklist. Available at https://tau.amegroups.com/ article/view/10.21037/tau-21-1011/rc

Peer Review File: Available at https://tau.amegroups.com/ article/view/10.21037/tau-21-1011/prf

Conflicts of Interest: All authors have completed the ICMJE uniform disclosure form (available at https://tau.amegroups. com/article/view/10.21037/tau-21-1011/coif). The authors have no conflicts of interest to declare.

Ethical Statement: The authors are accountable for all aspects of the work in ensuring that questions related to the accuracy or integrity of any part of the work are appropriately investigated and resolved. All procedures performed in this study were in accordance with the ethical standards of the institutional and/or national research committee(s) and with the Helsinki Declaration (as revised in 2013). Written informed consent was obtained from the patient for publication of this case report and accompanying images. A copy of the written consent is available for review by the editorial office of this journal.

Open Access Statement: This is an Open Access article distributed in accordance with the Creative Commons Attribution-NonCommercial-NoDerivs 4.0 International License (CC BY-NC-ND 4.0), which permits the noncommercial replication and distribution of the article with the strict proviso that no changes or edits are made and the original work is properly cited (including links to both the formal publication through the relevant DOI and the license). See: https://creativecommons.org/licenses/by-nc-nd/4.0/.

\section{References}

1. Hatemi G, Christensen R, Bang D, et al. 2018 update of the EULAR recommendations for the management of Behçet's syndrome. Ann Rheum Dis 2018;77:808-18. 
2. Giat E, Ehrenfeld M, Shoenfeld Y. Cancer and autoimmune diseases. Autoimmun Rev 2017;16:1049-57.

3. Lin Y, Li G, Zheng W, et al. Behcet's disease associated with malignancy: a report of 41 Chinese cases. Int J Rheum Dis 2014;17:459-65.

4. Ahn JK, Oh JM, Lee J, et al. Behcet's disease associated with malignancy in Korea: a single center experience. Rheumatol Int 2010;30:831-5.

5. Witjes JA, Bruins HM, Cathomas R, et al. European Association of Urology Guidelines on Muscle-invasive and Metastatic Bladder Cancer: Summary of the 2020 Guidelines. Eur Urol 2021;79:82-104.

6. Baltaci S, Gögüs C, Karamürsel T, et al. Invasive bladder carcinoma in a patient with Behçet's disease. Int J Urol 2003;10:669-71.

7. Sevinc C, Özkaptan O, Balaban M, et al. Radical Cystoprostatectomy in Patients with Behçet's Disease: The Report of Four Cases and Review of The Literature. Urol J 2016;13:2871-5.

8. Guo AT, Huang H, Wei LX. Clinicopathological characteristics and prognosis of Chinese patients with sarcomatoid carcinoma of the bladder. Histol Histopathol 2013;28:1167-74.

9. Brock N, Stekar J, Pohl J, et al. Acrolein, the causative factor of urotoxic side-effects of cyclophosphamide, ifosfamide, trofosfamide and sufosfamide. Arzneimittelforschung 1979;29:659-61.

10. Baker GL, Kahl LE, Zee BC, et al. Malignancy following treatment of rheumatoid arthritis with cyclophosphamide. Long-term case-control follow-up study. Am J Med 1987;83:1-9.

11. Sigal SH, Tomaszewski JE, Brooks JJ, et al. Carcinosarcoma of bladder following long-term cyclophosphamide therapy. Arch Pathol Lab Med 1991;115:1049-51.

12. Kurokawa MS, Yoshikawa H, Suzuki N. Behçet's disease. Semin Respir Crit Care Med 2004;25:557-68.

13. Seo J, Ahn Y, Zheng Z, et al. Clinical significance of serum YKL-40 in Behçet disease. Br J Dermatol 2016;174:1337-44.

14. Kim DY, Choi MJ, Kim HY, et al. Development and validation of an electronic medical record-based disease activity index for Behçet's disease. Clin Exp Rheumatol 2014;32:S40-4.

15. Calamia KT, Schirmer M, Melikoglu M. Major vessel involvement in Behçet's disease: an update. Curr Opin Rheumatol 2011;23:24-31.

16. Kalko Y, Basaran M, Aydin U, et al. The surgical treatment of arterial aneurysms in Behçet disease: a report of 16 patients. J Vasc Surg 2005;42:673-7.

17. Köklü S, Yüksel O, Onur I, et al. Ileocolonic involvement in Behçet's disease: endoscopic and histological evaluation. Digestion 2010;81:214-7.

18. Iscan ZH, Vural KM, Bayazit M. Compelling nature of arterial manifestations in Behcet disease. J Vasc Surg 2005;41:53-8.

19. Xue X, Wang D, Ji Z, et al. Risk factors of postoperative ileus following laparoscopic radical cystectomy and developing a points-based risk assessment scale. Transl Androl Urol 2021;10:2397-409.

20. Bhatti S, Malik YJ, Changazi SH, et al. Role of Chewing Gum in Reducing Postoperative Ileus after Reversal of Ileostomy: A Randomized Controlled Trial. World J Surg 2021;45:1066-70.
Cite this article as: Xue X, Yang X, Ji Z, Xie Y. Case report: laparoscopic radical cystectomy on a patient with Behçet's syndrome, our initial experience on perioperative safety. Transl Androl Urol 2022;11(2):285-292. doi: 10.21037/tau-21-1011 